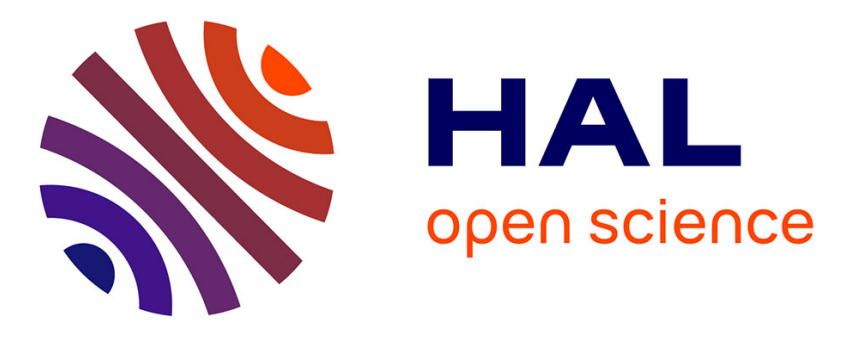

\title{
3D harnessing of light with photon cage
}

Clément Sieutat, Jean-Louis Leclercq, Xavier Letartre, Ségolène Callard, M. Gendry, Grenet Geneviève, Khalid Naji, Philippe Regreny, Pedro

Rojo-Romeo, P. Viktorovich, et al.

\section{- To cite this version:}

Clément Sieutat, Jean-Louis Leclercq, Xavier Letartre, Ségolène Callard, M. Gendry, et al.. 3D harnessing of light with photon cage. SPIE Photonics Europe 2010, Apr 2010, Bruxelles, Belgium. pp.77120E, 10.1117/12.854428 . hal-00692495

\section{HAL Id: hal-00692495 \\ https://hal.science/hal-00692495}

Submitted on 30 Apr 2012

HAL is a multi-disciplinary open access archive for the deposit and dissemination of scientific research documents, whether they are published or not. The documents may come from teaching and research institutions in France or abroad, or from public or private research centers.
L'archive ouverte pluridisciplinaire HAL, est destinée au dépôt et à la diffusion de documents scientifiques de niveau recherche, publiés ou non, émanant des établissements d'enseignement et de recherche français ou étrangers, des laboratoires publics ou privés. 


\title{
3D harnessing of light with photon cage
}

\author{
C. Sieutat, J.L. Leclercq, X. Letartre, S. Callard, M. Gendry, G. Grenet, , K. Naji, P. Regreny, P. \\ Rojo-Romeo, P. Viktorovitch
}

Université de Lyon, Institut des Nanotechnologies de Lyon (INL), UMR CNRS 5270

Ecole Centrale de Lyon, 36 avenue Guy de Collongue, F 69134 Ecully Cedex, France

Contact jean-louis.leclercq@ec-lyon.fr

G. Beaudin, M. Cloutier, D. Drouin, V. Aimez

Centre de Recherche en Nanofabrication et en Nanocaractérisation $\left(\mathrm{CRN}^{2}\right)$, Université de Sherbrooke, $\mathrm{CRN}^{2}, 2500$ boulevard Université, Sherbrooke, QC, J1K 2R1, Canada

\begin{abstract}
We report on design, simulation and fabrication of ultimate and compact 3D close-geometries optical microcavities. These are based on the extension of the so-called 2.5D nanophotonic approach where a quasi 3D control of the photons has been soon demonstrated by our group. A tight control of photons, spectrally and spatially, in a small air region inside a circular regular pattern of high index material-based nanopillars is demonstrated when adjusting the number of pillars, their diameters and the diameter of the pillar-circle. Bottom-up approach based on InP nanowires grown by molecular beam epitaxy and top-down approach based on high aspect ratio anisotropic etching have been developed for fabricating these optical microcavities.
\end{abstract}

Keywords: 3D Photonic crystal, optical microcavity, microresonator, nanophotonics

\section{INTRODUCTION}

Micro-Nano-Photonics can be defined as the control of photons within the tiniest possible space during the longest possible time. The generic conceptual and technological brick is the optical microresonator. This presents the ability to confine photons within tiny volumes which is essential for the efficient operation of a wide range of active micro-nanophotonic devices, including low threshold micro-lasers and non-linear optic devices.

In the same way, tight control of the emission diagram can be realized thus yielding to propose new optical functionalities such as collection, cascability, routing...The general approach to achieve strong confinement of photons consists in high index contrast structuring of space at the wavelength scale. In this regards, multiple demonstrations have been made with microdisk-, micropillar- and one- or two-dimensional (1D - 2D) photonic crystals-based microcavities. All these have been developed using well-controlled planar technological approaches.

Experimental demonstrations have shown their relative limitation of the final confinement range. Three-dimensional (3D) photonics crystals can block photons in any direction and are expected to make possible their ultimate control. However, their practical fabrication over large areas without any defects is extremely complex and can be considered as challenging. In our group, significant progress for quasi 3D control of photons have been achieved along a 2.5D nanophotonic approach which can be considered as an extension of planar 2D photonic crystal exploiting the vertical

Nanophotonics III, edited by David L. Andrews, Jean-Michel Nunzi, Andreas Ostendorf, Proc. of SPIE Vol. $7712,77120 \mathrm{E} \cdot$ @ ( 2010 SPIE · CCC code: 0277-786X/10/\$18 - doi: 10.1117/12.854428 
direction $^{1-8}$. Today we propose a new concept of trapping photons in real $3 \mathrm{D}$ optical microresonators that can be assimilated to 'photon cages'. It is mainly a question of exploring and of combining new scientific and technological concepts based on engineering of optical modes and micro-nanostructuring to generate objects of various closed geometries at the wavelength scale in order to achieve a real 3D control of the light.

This new optical configuration allows to artificially control and drastically enhance the electromagnetic field in the central part of the cage in the air region yielding to new sensing or trapping of nanoparticles in fluidic (gas or liquid) ambiances.

\section{PHOTON CAGE BASIC PRINCIPLES: FROM 2,5D TO 3D}

PC-based photonic devices operating in the waveguided configuration have been principally aimed so far at forming the basic building blocks of integrated photonics and have been the topics of considerable amount of research worldwide, resulting in an ever growing number of new device demonstrations, so much so that it may be stated today that PCs have entered within the realm of practical devices.

Optical losses, which result from the unwanted coupling of waveguided modes with radiated modes are however considered as a major drawback of PC-based photonic integration and restrict the operation of the devices below the socalled light line, where this coupling is theoretically impossible. This leaves not much room in the (frequency, wave vector) or $(\omega, k)$ space for a lossless operation of the devices. In that respect, the constraints are less stringent in the socalled membrane approach, where the waveguide slab is formed by a high-index dielectric layer (e.g., semiconductor layer) embedded in low-index cladding layers. This approach has been adopted by most groups in the field. The 2D PC formed in planar waveguide slabs are far more accessible than 3D PC, since they may be fabricated using planar technological schemes, which are familiar to the world of integrated optics and microelectronics. They result in more compact structures.

The problem of optical losses, however, can be approached from a completely different perspective. Instead of attempting to confine the light entirely within the waveguide structures, the latter can be deliberately opened to the third space dimension by controlling the coupling between waveguided and radiation modes. In this case, the exploitation of the optical power is achieved by accurately tailoring the optical radiation into free space. The operation of the devices is based on the resonant coupling between radiated optical modes and slow Bloch modes waveguided in the membranes, which are laterally patterned to form a PC (figure 1). Along this line, a new class of compact surface addressable structures using PC in the waveguided configuration has been proposed in the recent literature and has given rise to a flourishing production of devices including compact wavelength selective reflectors ${ }^{2}$, surface emitting microlasers ${ }^{3-6}$ or nonlinear devices ${ }^{7-8}$. It has been proposed recently that a major extension of planar technology through exploitation of the third ("vertical") dimension by using a so-called multilayer approach, where the lateral high-index contrast patterning of layers would be combined with a vertical 1-D index contrast patterning.

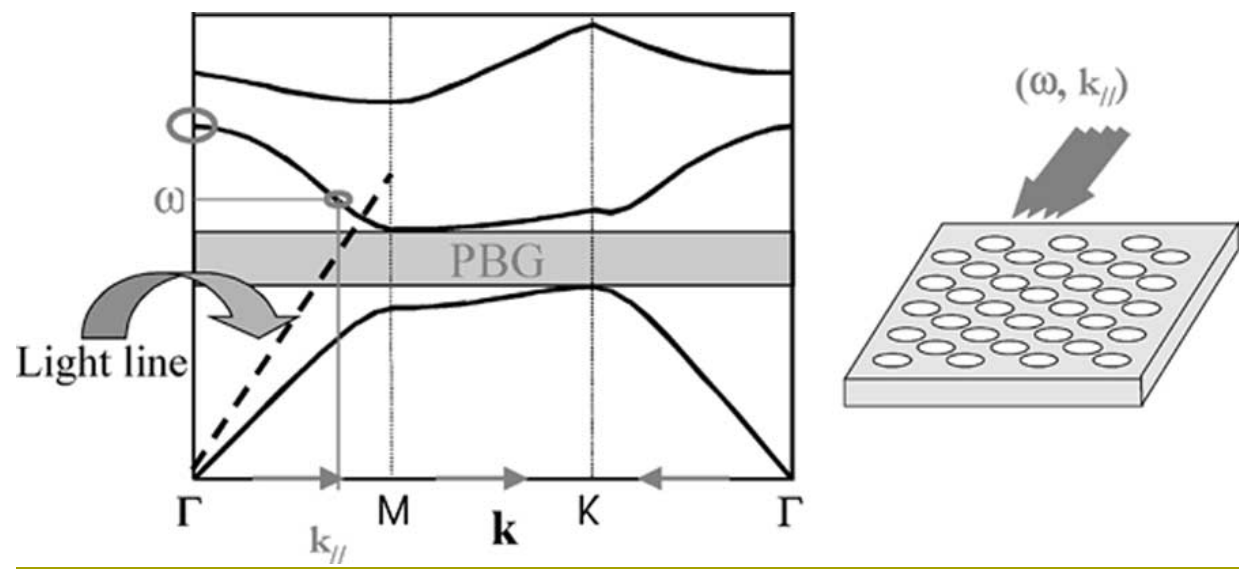

Figure 1 : Illustration of the resonant coupling between a waveguided mode and a radiative mode 
Here, it is more appropriate to think in terms of "2.5-dimensional" photonic structures which can be considered as an intermediate step between 2D and 3D. 2.5D PC have been shown to provide a quasi-3D harnessing of the light at the wavelength scale, via the controllable interplay occurring between waveguided confined photons and radiated photons propagating through the planar multilayer structure, at a far lower technological cost than pure 3D PC.

Novel class of highly efficient and broadband mirrors have been recently proposed using single-layer high transverseindex contrast photonic crystal mirrors $(\mathrm{PCM})^{9-11}$. These single-layer PCM are able to provide very efficient and broadband reflectivity with a very small thickness. Vertical Fabry Perot cavities (VFPC) have been extensively studied, especially for the realization of vertical-cavity surface emitting lasers (VCSELs). These are traditionally composed of two Distributed Bragg Reflectors (DBR) which reflectivity has to be sufficient in order to obtain highly resonant cavity, which is particularly necessary for laser emission in VCSELs. As a consequence, DBRs consist generally in very thick layer stacks. We demonstrated the smallest conceivable high Q vertical Fabry-Perot cavity, using ultra-thin and highlyefficient photonic crystal slab mirrors instead of conventional DBRs (see figure 2 and 3$)^{11}$. Use of two PCM improves significantly the vertical compactness of the filter layers. The selectivity of the filter is, to a large extent, controlled by the lateral escape rate of photons out of the PCM area or, in other words, by the time that it takes to the hybrid resonant optical mode to extend over an area exceeding to that of the PCM.

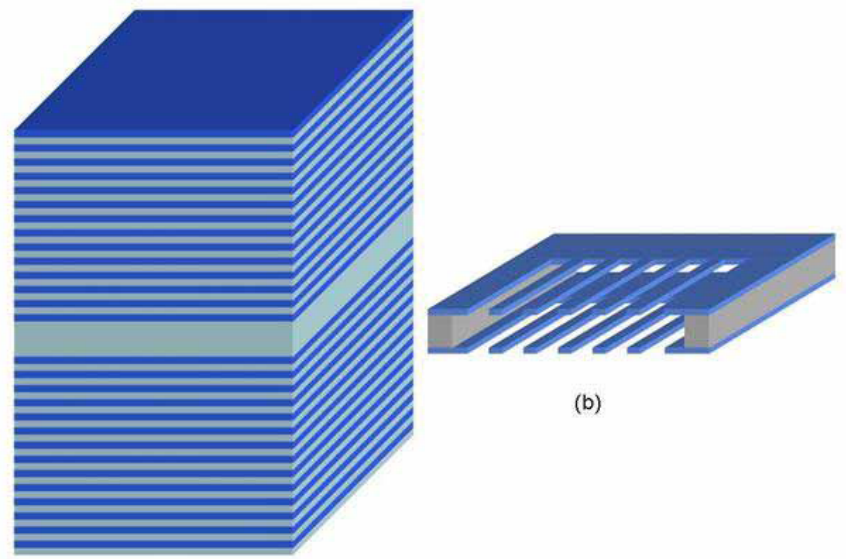

(a)

Figure 2 : Schematic representation of a classical DBR-VFPC (a) and a PCM-VFPC (b)

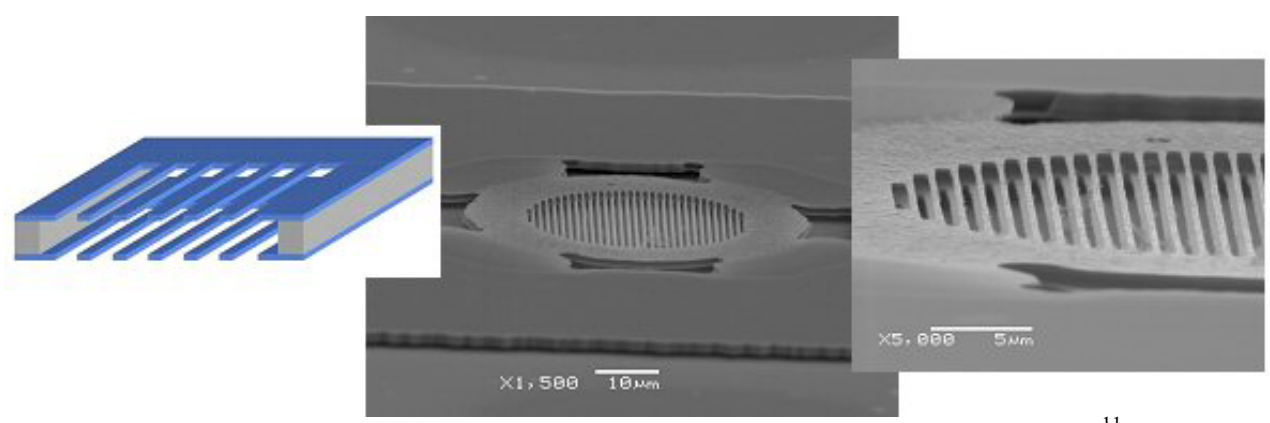

Figure 3 : Schematic and experimental realisation of InP-based PCM-VFPC ${ }^{11}$

Now, we propose to extend the $2.5 \mathrm{D}$ concept to real 3D directly by generating $3 \mathrm{D}$ microcavities presenting closed geometries deviating from these ultimate photonic crystal mirrors-based vertical Fabry-Perot microcavities. These 'photon cages' can be seen as 'physical' deformation of the geometry of the vertical microcavity as shown schematically in figure 4. 


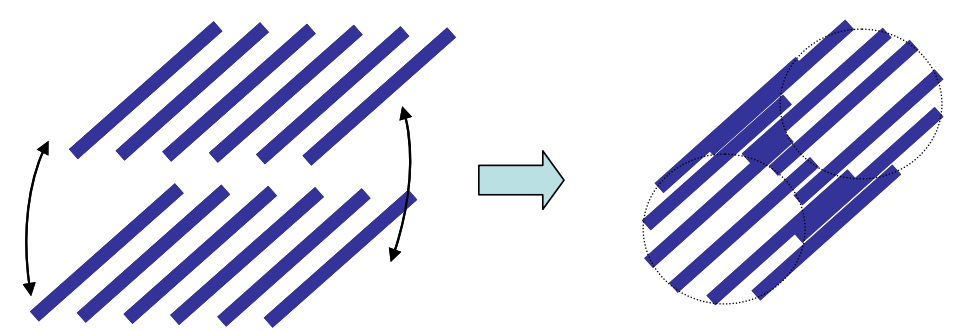

Figure 4 : Schematic representation of the concept 'from 2.5D to 3D' microcavity

By generating these micro-objects with various closed geometries (spires, tubes, rolls...) at wavelength scales combined with dedicated optical modes engineering in the low index parts (air region) it may yield to tight control of the photons spatially and spectrally. Since these objects are mainly composed by air, their ability to sense or trap nanoparticles which can interfere with the cage resonant modes are drastically enhanced. Moreover, if we are able to induce directly on the dimensions of the object (mechanical, optical and/or electrical modification), efficient modulation (tuning) of the optical properties may be achieved.

\section{DESIGN OF A PHOTON CAGE}

The photon cage used in our approach can be illustrated schematically as presented in figure 5. This is based on a vertical regular circular array of high index semiconductor material nanopillars with high aspect ratio.

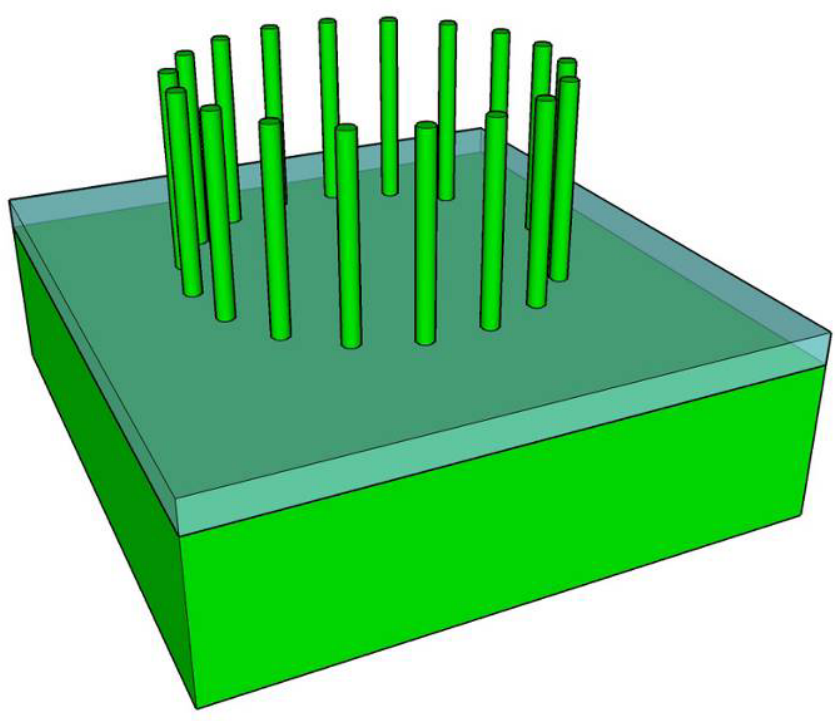

Figure 5 : Schematic 3D representation of the photon cage

The design of this "cage" can be made by considering in a first step the reflectivity characteristics of a PCM composed of a 1D periodic array of $\mathrm{Si}(\mathrm{n}=3,5)$ pillars in air (figure 6$)$. The reflection spectrum is calculated by 2D FDTD for light polarization parallel to the pillars axis. Optimization of the parameters (pillars diameter D, period a) leads to a broadband mirror with reflectivity larger than $99.5 \%$ in the $1500-1600 \mathrm{~nm}$ wavelength range. 


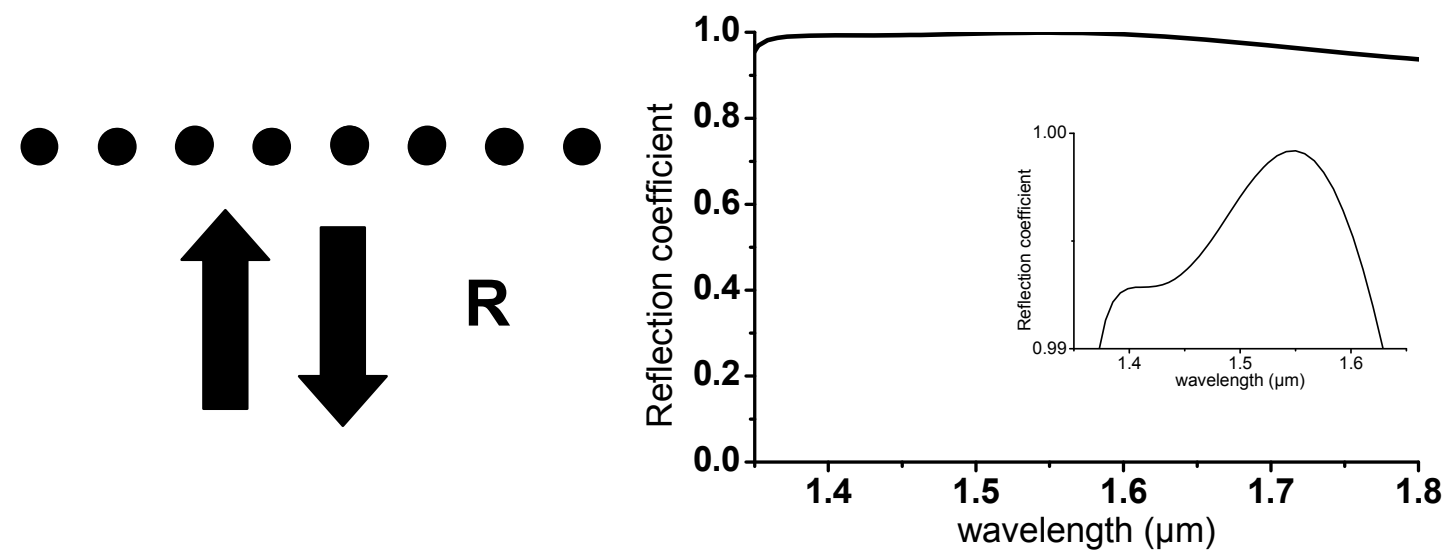

Figure 6 : Theoretical reflectivity spectra of a $1 \mathrm{D}$ array of a periodic array of Si pillars. The parameters are $\mathrm{a}=1 \mu \mathrm{m}, \mathrm{D}=0.27 \mu \mathrm{m}$.

This linear 1D PC structure is then 'deformed' in order to obtain a circular cage of pillars with the same period (a=1 $\mu \mathrm{m})$ along the circle delimiting the cage. Therefore, the only adjustable parameter is the number of pillars, N. 2D FDTD calculations are used, assuming pillars with infinite height, to characterized the resonant modes of such a structure. Due to its quasi cylindrical nature, this cavity exhibits Whispering Gallery-like modes which are affected by the periodic corrugation at the circular boundary (see examples on figure 7).
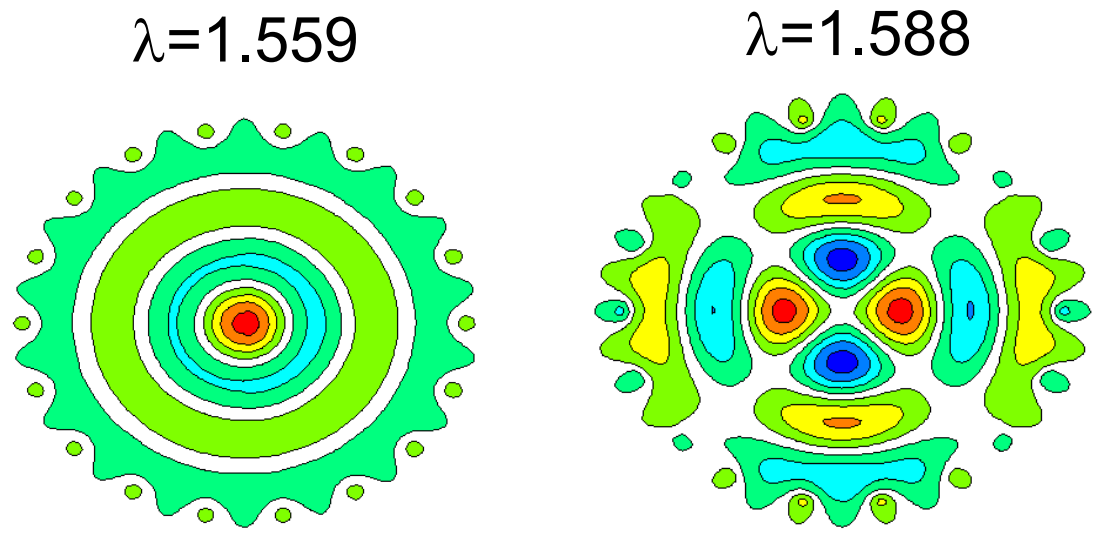

Figure 7 : Electric field distribution for two resonant modes of a $\mathrm{N}=18$ cage

As we aim at confining the electromagnetic energy in the middle of the air cavity, we focus our design on the "radial" mode (left of figure 7, corresponding to an azymuthal order $\mathrm{m}=0$ in the conventional WGM classification). The highest quality factor, $\mathrm{Q}=7000$, is obtained for a cage with $\mathrm{N}=18$. Then, a slight modification of the pillar diameter, from $\mathrm{D}=0.27 \mu \mathrm{m}$ to $\mathrm{D}=0.26 \mu \mathrm{m}$ (less than $4 \%$ ), leads to $\mathrm{Q}=5 \times 10^{4}$.

It is worthwhile to notice that an extremely high concentration of the field is reached at the center of the cage, although the cavity is composed of more than $95 \%$ of air. More specifically, we calculate a mode surface, defined as the inverse of the normalized field intensity maximum, of $0.7 \lambda^{2}$. when the cavity surface is about $11 \lambda^{2}$.

3D FDTD calculations for a finite structure with a $6 \mu \mathrm{m}$ height show a drastic reduction of the quality factors (Q 600$)$, indicating strong vertical optical losses. Despite this quite low value, a very good confinement is obtained. Indeed, considering the Purcell effect as a factor of merit, we get: 


$$
F_{P}=\frac{3}{4 \pi^{2}} \frac{\lambda^{3}}{V} Q \approx 40
$$

To a certain extent, this photons cage can be viewed as a vertical planar microcavity where the radial direction takes place of the vertical one. Then, it is straightforward to show, by considering the $k_{Z}$ dispersion of the mode, that $F_{P}$ should be proportional to the height of the cage. This has to be confirmed by additional 3D FDTD simulations.

These first theoretical calculations show that it should be very important to fabricate very high aspect ratio vertical circular array of nanopillars.

\section{TECHNOLOGICAL APPROACHES}

Today, nanosize vertical semiconducting material structures with high aspect ration are crucial for many applications. Two approaches are generally followed to fabricate them and more specifically nanowires (NWs). The first relies on bottom-up approach based on catalyst-assisted growth of vertical nanowires by Vapor Liquid Solid (VLS) mechanism ${ }^{12}$. The second one relies on top-down manufacturing using high-aspect-ratio anisotropic etching. Both of these technological approaches are well known and are constantly competiting for producing single nanofabrication standard.

Great efforts have been made to control NWs growth directions and to enhance vertical orientation by molecular beam epitaxy (MBE) ${ }^{13-14}$. Many groups have obtained vertically oriented III-V NWs on Si(111) because the NWs grow preferentially in the $<111>$ directions. In our group, we developed InP NWs growths on $\mathrm{Si}(001), \mathrm{STO}(001)$ and $\mathrm{InP}(111)$ substrates $^{15}$. For our project, first tentative of fabricating photon cages on the bottom-up approach has been performed by MBE. InP NWs were grown by VLS assisted solid source MBE at a growth temperature of $380^{\circ} \mathrm{C}$ with $\mathrm{Au}$ as catalyst metal on $\mathrm{InP}(111)$ substrates. $40 \mathrm{~nm}$ thick $\mathrm{Au}$ dot patterns are previously deposited by a combined ebeam lithography writing and metal lift-off procedure (figure 8).

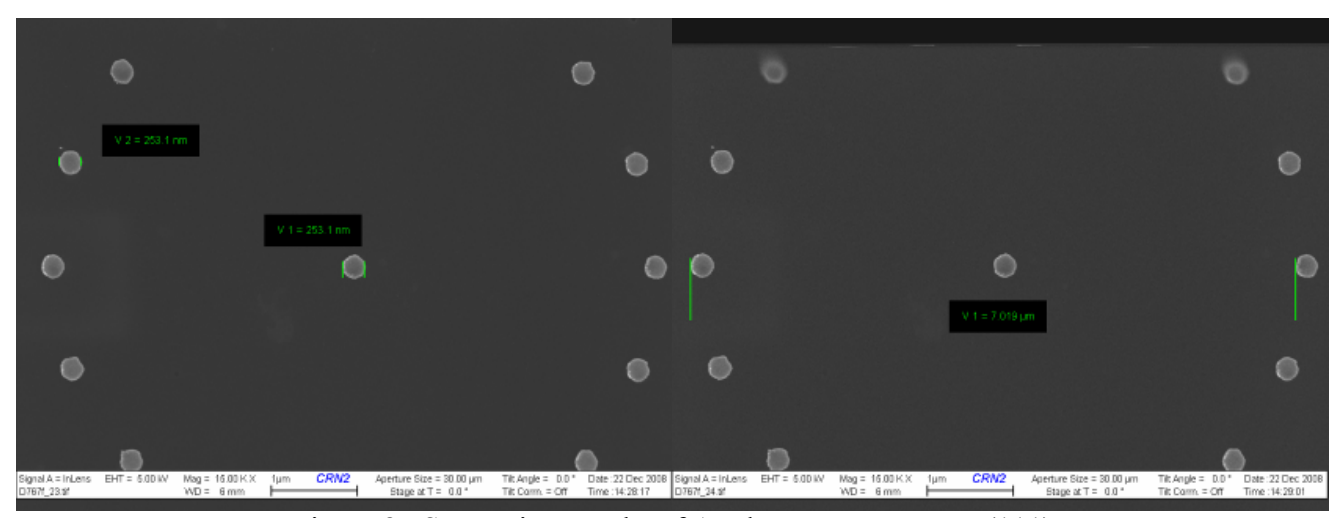

Figure 8 : SEM micrographs of Au dot-patterns on InP (111)

(diameters of about $253 \mathrm{~nm}$ for the dots and $7 \mu \mathrm{m}$ for the cage)

Figure 9 shows a single cage of $1 \mu \mathrm{m}$ height InP NWs grown on InP (111). We note that some NWs have grown parasitically, we believe that this is a consequence of not well-defined Au dot pattern by lift-off leaving on the surface additional metal particles. Moreover, we note that height and width of the InP NWs have to be still optimized. 


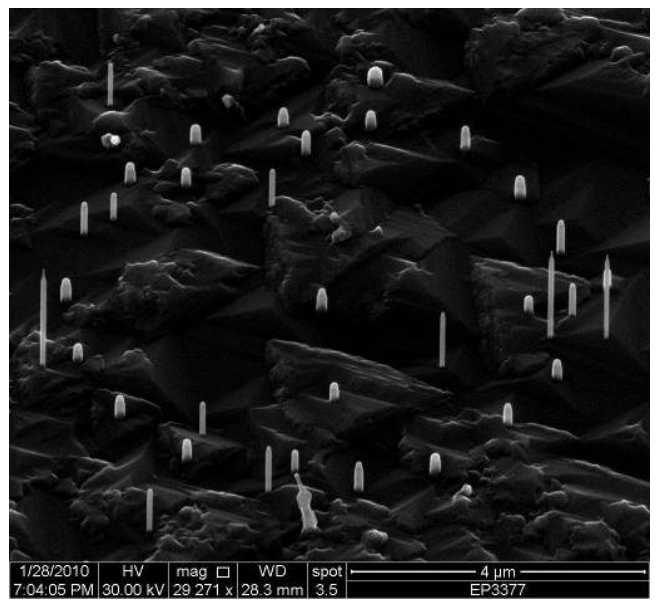

Figure 9 : SEM view of InP NWs grown by VLS-MBE on InP (111)

For the top-down approach, deep reactive ion etching (DRIE) is investigated for the realization of the nanostructures. Double side polished silicon-on-insulator (SOI) with $10 \mu \mathrm{m}$ device layer and $3 \mu \mathrm{m}$ buried oxide (BOX) was selected. After cleaning, the $\mathrm{SiO}_{2}$ and chromium hard masks were deposited by plasma-enhanced chemical vapor deposition (PECVD) and e-beam evaporation respectively. Submicron dot patterns of various size and diameters (figure 10) were then prepared by e-beam lithography via spin coated negative electro-resist.

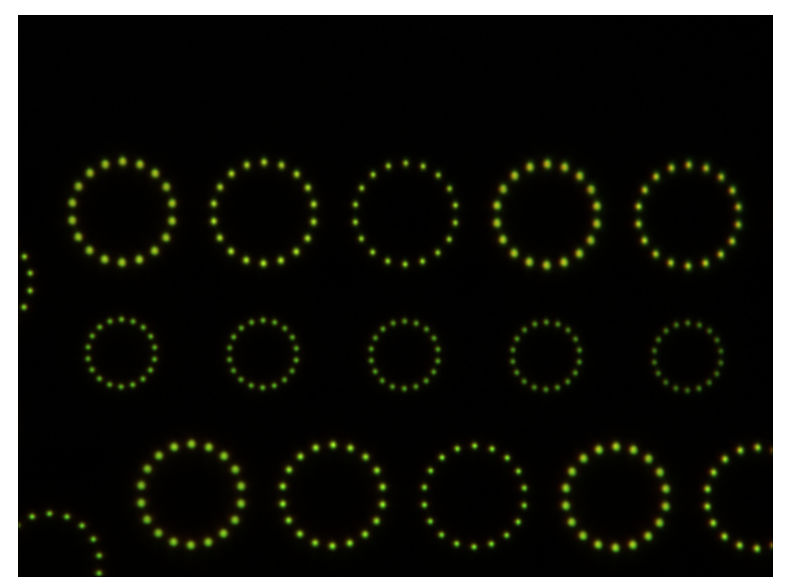

Figure 10 : Dark field picture of submicron dots-cavities for mask patterning

The exposition and development are followed by three inductively-coupled plasma (ICP) etching steps. The first one is the transfer of the resist pattern to the chromium by using a $\mathrm{Cl}_{2}-\mathrm{He}-\mathrm{O}_{2}$ recipe. Then, the second one is the transfer from the chromium to the $\mathrm{SiO}_{2}$ with $\mathrm{CF}_{4}$ chemistry with an excellent accuracy and reproducibility (figure 11). 


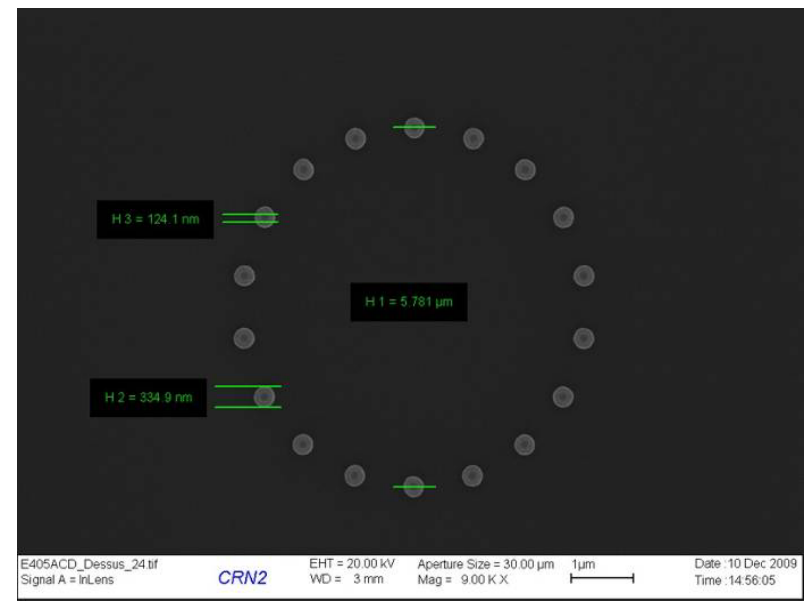

Figure $11: \mathrm{Cr} / \mathrm{SiO} 2$ mask pattern transferred by ICP on SOI (diameters of about $334 \mathrm{~nm}$ for dots and $5.78 \mu \mathrm{m}$ for the cage)

Finally, the silicon from the device layer of the SOI is etched all through the BOX by Bosch process. The etching use $\mathrm{SF}_{6}-\mathrm{C}_{4} \mathrm{~F}_{8}-\mathrm{O}_{2}$ chemistry and the passivation use $\mathrm{C}_{4} \mathrm{~F}_{8}$ only. Using this dedicated triple-cycling etching procedure, we produced with great success $10 \mu \mathrm{m}$ height Si pillar-based cavities with different densities as shown in figure 12 .

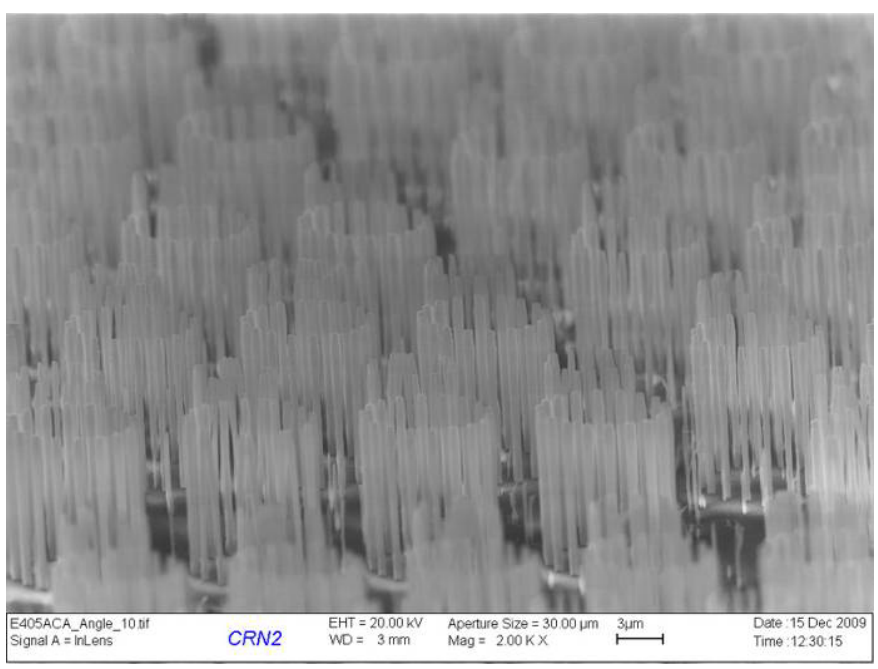

Figure 12 : Dense forest of Si pillar-based circular cavities

\section{CONCLUSION}

In summary, we have demonstrated that the quasi-3D optical confinement based on the $2.5 \mathrm{D}$ approach can be theoretically and experimentally extended to a real 3D configuration. Design and simulation of the deformation of a linear 1D PCM into a 2D circular dots-based reflector have shown highly resonant modes located perfectly at the centre of this new cavity. Comparing to microdisk-, micropillar- or 1D - 2D photonic crystals optical microcavities, the optical modes of such 3D microstructures should present great differences due to their particular geometries (e.g. along the vertical axe of the cage).

Experimental works for producing these 3D cavities have been performed by bottom-up and top-down approaches with very promising results. If the first approach needs more optimization studies for growing by VLS high aspect ratio pillar 
arrays, the second one based on DRIE is now more close to the validation of the concept. SNOM-AFM optical measurements are now envisaged for mapping the optical modes and for demonstrating the $3 \mathrm{D}$ harnessing of the light with the photon cage.

A third explorative approach, also developed in our group ${ }^{\mathrm{i}}$, is based on the concept of self-rolling strained multilayers to created 3D novel microcavities of various controllable shapes (nanotubes, nanospirals, rings, cylinders...) ${ }^{16}$.

Such novel 3D confinement in the air will produce a new class of closed nanophotonic devices for chemical and biochemical sensing applications.

\section{ACKNOWLEDGEMENTS}

This work is a part of a common project into the frame of the International Laboratory on Nanotechnologies and Nanosystems between CNRS in France and University of Sherbrooke in Canada (http://www.labn2.ca). Authors are gratefully acknowledging technological staffs from Nanolyon and $\mathrm{CRN}^{2}$ platforms. Region Rhône-Alpes is also acknowledged for its financial support through the PhD grant of C. Sieutat.

\section{REFERENCES}

[1] Letartre X. , Mouette J., Seassal C., Rojo-Romeo P., Leclercq J.L., Viktorovitch P., "Switching devices with spatial and spectral resolution combining photonic crystal and MOEMS structures", Journal of Lightwave Technology, 21, 7, 1691-1699 (2003)

[2] Boutami S., Ben Bakir B., Hattori H., Letartre X., Leclercq J.L., Rojo Romeo P., Garrigues M., Seassal C., Viktorovitch P., "Broadband and compact 2-D photonic crystal reflectors with controllable polarization dependence," IEEE Photon. Technol. Lett., 18 , no. 5-8, 835-837 (2006)

[3] Ben Bakir B., Seassal C., Letartre X., Viktorovitch P., "Surface emitting microlaser combining 2D photonic membrane and 1D vertical Bragg mirror,” Appl. Phys. Lett., 88, 081113 (2006)

[4] Boutami S., Ben Bakir B., Leclercq J.L., Viktorovitch P., "Compact and polarization controlled 1,55 $\mu \mathrm{m}$ vertical-cavity surface emitting laser using single-layer photonic crystal mirror”, Applied Phys. Lett., 91, 071105 (2007)

[5] Ferrier L., Rojo-Romeo R., Drouard E., Letartre X., Viktorovitch P., "Slow Bloch mode confinement in 2D photonic crystals for surface operating devices", Optics Express, 16, Issue 5, 3136-3145 (2008)

[6] Mouette J., Seassal C., Letartre X., Rojo Romeo P., Leclercq J.L., Regreny P., Viktorovitch P., Jalaguier E., Perreau P., Moriceau H., "Very low threshold vertical emitting laser operation in InP graphite photonic crystal slab on silicon," Electron. Lett., $39(6), 526-528(2003)$

[7] Cojocaru C., Raineri F., Monnier P., Seassal C., Letartre X., Viktorovitch P., Levenson A., Raj R., "Ultrafast nonlinear dynamics of a two-dimensional InP-based photonic crystal response,” Appl. Phys. Lett., 85, 1880 (2004).

[8] Yacomotti A.M., Ben Bakir B., Raineri F., Vecchi G., Monnier P., Letartre X., Seassal C., Viktorovitch P., Raj R., Levenson A., "All optical bi-stable slow Bloch modes in a two-dimensional photonic crystal", Appl. Phys. Lett., vol. 88, 231107 (2006)

[9] Mateus C.F.R., Huang M.C.Y., Chen L., Chang-Hasnain C.J., Suzuki Y., "Broadband mirror (1.12-1.62 $\mu \mathrm{m})$ using single-layer sub-wavelength grating," IEEE Photon. Technol. Lett., 16, 1676-1678 (2004)

[10] Lousse V., Suh W., Kilic O., Kim S., Solgaard O., Fan S., “Angular and polarization properties of a photonic crystal slab mirror," Opt. Express, 12, 1575-1582 (2004)

[11] Boutami S., Ben Bakir B., Letartre X., Leclercq J.L., Regreny P., Viktorovitch P., "Ultimate vertical Fabry-Perot cavity based on single-layer photonic crystal mirrors", Optics Express, 15, Issue 19, 12443-12449 (2007)

[12] Pauzauskie P. J., Yang P., "Nanowires photonics", Materials Today, 9, 36-45 (2006)

[13] Tomioka K., Motohisa J., Hara S., Fukui T., "Control of InAs nanowire gr owth direections on Si”, Nano Letters, 8, 3475$3480(2008)$

[14] Boles S.T., Thompson C.V., Fitzgerald E.A., "Influence of indium and phosphine on Au-catalyzed InP nanowire growth on Si substrates", Journal of Crystal Growth, 311 (5), 1446-1450 (2009)

[15] Naji K., Dumont H., Saint-Girons G., Robach Y., Rojo-Romeo P., Bru-Chevallier C., Gendry M., Patriarche G., "InP nanowires grown on Silicon and SrTiO3 by VLS assisted MBE", Proceedings IPRM 2008, May 25-29, 2008, Versailles (France)

[16] Prinz V.Y., S.V. Golod, "elastic silicon-film-based nanoshells : formation, properties and applications", J. of Appl. Mecha. And Technical Phys., 47 (6), 867-878 (2006)

\footnotetext{
i « ORIGAMI » project granted by the Region Rhône-Alpes CIBLE 2009 program
} 